\title{
¿CÓMO MEDIR LA COMPETITIVIDAD DE LAS MIPYMES?
}

\section{¿HOW TO MEASURE THE COMPETITIVENESS OF THE MIPYMES?}

Jesús Amador Valdés Díaz de Villegas Doctor en Ciencias Económicas por la Universidad de la Habana y Maestro en Economía y Política Internacional, Profesorinvestigador, Dpto. de Estudios Empresariales, Universidad Iberoamericana jesus.valdes@ibero.mx

\section{Resumen}

Cuando se habla de competitividad se hace referencia a la capacidad que tienen algunas empresas de alcanzar resultados y posiciones exitosas en su entorno socioeconómico durante un período sostenido de tiempo, a partir del desarrollo y mantenimiento de sus ventajas competitivas en el entorno socioeconómico. Es un término que no tiene determinación cuantitativa, se trata de poseer una posición defendible con ventajas sostenibles.

Las MIPYMES han recibido poca atención en relación a la competitividad, en los países en vías de desarrollo a pesar de su importancia. En México la estructura empresarial está compuesta en su mayoría por micros y pequeñas empresas, que conforman más del $95.2 \%$ del total de la industria, esto demuestra la importancia que reviste este tipo de empresas, que conforman una parte 
fundamental en los procesos de recuperación y de reordenación de la economía nacional y en el cambio estructural del aparato productivo que el país requiere.

Nuestro objetivo en este trabajo es presentar una metodología que constituya la base para poder medir en nuestras condiciones la competitividad de las micro, pequeñas y medianas empresas. Por tanto presentamos las bases de lo que hemos denominado el Índice lbero de Competitividad Empresarial.

\section{Abstract}

When talking about competitiveness refers to the ability of some companies to achieve successful results and positions in its socioeconomic environment for a sustained period of time, from the development and maintenance of its competitive advantages in the socioeconomic environment. It is a term that has no quantitative determination; this is a defensible position to possess sustainable advantages.

MSMEs have received little attention in relation to competitiveness in developing countries despite its importance. In Mexico the company structure consists mainly of micro and small businesses, which make up more than $95.2 \%$ of the total industry, this demonstrates the importance of these businesses, which make up a 
major part in the recovery process and rearrangement of the national economy and the structural change of the production that the country needs.

Our aim in this paper is to present a methodology that is the basis to measure in our conditions the competitiveness of micro, small and medium enterprises. Therefore we present the foundations of what we have called the Business Competitiveness Index Ibero.

\section{Introducción}

La empresa es la célula básica para la producción de bienes y servicios, constituye una unidad económicosocial con fines de lucro, en esta unidad los factores de producción se coordinan y conjugan para general la creación de bienes de interés social e individual. Los elementos necesarios para formar una empresa son: capital, trabajo, tierra y habilidades empresariales. En general, se entiende por empresa al organismo social integrado por elementos humanos, técnicos y materiales cuyo objetivo natural y principal es la obtención de utilidades, o bien, la prestación de servicios, coordinados por un administrador que toma decisiones para que se puedan lograr las finalidades establecidas en su constitución.

En el ejercicio de su actividad económica, la empresa moderna produce indudables beneficios sociales. En general, proporcionan al público un abastecimiento oportuno y adecuado y una distribución más efectiva de bienes y servicios. A través de la difusión del crédito, ha incrementado la capacidad de compra de grandes 
sectores de la población y, por medio de la publicidad, han llevado el conocimiento de nuevos productos capaces de satisfacer sus necesidades sociales e individuales. Todos los beneficios generados por la empresa moderna, se han visto apoyados por el aumento en la productividad, que ha provocado un crecimiento de la eficiencia y aumentando la producción en masa generando un proceso de disminución de precios. Sin embargo, la empresa moderna no solo debe cumplir con sus finalidades económicas. La gente, en general, espera que las empresas tomen parte también en otras áreas de la vida social y aporte soluciones.

La empresa, como célula básica del sistema de generación de bienes y servicios, es la institución clave de la vida económica, que permite la manifestación de la creatividad y la libertad jurídica de las personas. Esencialmente, es un grupo humano al que unos hombres le aportan capital, otros, trabajo y otros aportan sus habilidades de dirección, para conseguir determinadas finalidades económicas.

La empresa, es una identidad endógena a la sociedad y no puede permanecer ajena a ella. La sociedad le proporciona a las empresas la paz y el orden que debe ser garantizado por la ley y el poder público; la fuerza de trabajo y el mercado de consumidores; la educación de sus obreros, técnicos y directivos; los medios de comunicación y la llamada infraestructura económica. Por eso no puede decirse que las finalidades económicas de la empresa estén por encima de sus finalidades sociales. Ambas están también indisolublemente ligadas entre sí y 
se debe tratar de alcanzar unas, sin detrimento 0 aplazamiento de las otras.

En lo anterior descansa la responsabilidad social empresarial, el rol que la empresa tiene para con la sociedad, que va más allá de la simple producción y comercialización de bienes y servicios. La empresa, también tiene que asumir compromisos con los grupos de interés existente en la sociedad para contribuir a solucionar problemas: como pobreza, medio ambiente, etc. Una enumeración de los beneficios concretos y tangibles que significa para la empresa es mantener en alto su responsabilidad social, por tanto es necesario:

- Un incremento de la productividad, porque el trabajador está a gusto en la empresa y se le capacita para que lo haga cada vez mejor;

- Mejoramiento de las relaciones con los trabajadores, el gobierno y con las comunidades a nivel regional y nacional;

- Un mejor manejo en situaciones de riesgo o de crisis, ya que se cuenta con el apoyo social necesario;

- Sustentabilidad en el tiempo para la empresa y para la sociedad, dado que la responsabilidad social fortalece el compromiso de los trabajadores, mejora su imagen corporativa y la reputación de la empresa, entre otros.

- Imagen corporativa y reputación, frecuentemente los consumidores son llevados hacia marcas y compañías consideradas por tener una buena reputación en áreas relacionadas con la responsabilidad social empresarial. También importa en su reputación entre la comunidad empresarial, incrementando así la habilidad de la empresa para 
atraer capital y asociados, y también con los empleados dentro de la empresa.

- Rentabilidad de sus negocios. Se ha demostrado que las empresas fieles a sus códigos de ética resultan de un desempeño de dos a tres veces superior respecto a aquellas que no los consideran, de esta forma las compañías con prácticas socialmente responsables obtienen tasas de retorno a sus inversiones muy superiores a las expectativas.

- Reducción de Costos Operativos, son múltiples las iniciativas que logran reducir costos a las empresas, principalmente del área ambiental, como los es el reciclaje, que genera ingresos extras.

- Acceso al Capital, las compañías que demuestran responsabilidades éticas, sociales y medioambientales tienen acceso disponible a capital, que de otro modo no hubiese sido sencillo obtener.

A todo lo anterior hay que unir que la empresa que tiene la cualidad de la competitividad, la capacidad y habilidad para competir exitosamente en su mercado. La empresa competitiva es una organización en la cual todos sus componentes están alineados para tener la capacidad para producir y mercadear sus productos, bienes 0 servicios, en mejores condiciones de precio, calidad y oportunidad que sus rivales.

En este artículo es presentar una metodología que constituya la base para poder medir en condiciones de nuestra estructura organizativa empresarial la competitividad de las micro, pequeñas y medianas empresas. Por tanto se presentan las bases de lo que 
hemos denominado el Índice Ibero de Competitividad Empresarial.

\section{Medición de la Competitividad Empresarial por el Fórum Mundial}

A partir del año 2000, el Informe de Competitividad Global del Fórum Económico Mundial, se le integraron dos enfoques complementarios para el análisis de la competitividad:

1. Índice de Competitividad para el crecimiento (Growth Competitveness Index), que mide la capacidad de la economía nacional, para lograr un crecimiento económico sostenido en el mediano plazo y se basa en tres amplias categorías: tecnología, instituciones públicas y ambiente macroeconómico.

2. Índice de Competitividad para los Negocios (Business Competitiveness Index o $\mathrm{BCl}$ (denominación que se utiliza a partir de 2003, ya que anteriormente se conocía como Índice de Competitividad Microeconómica o $\mathrm{MICl}$ ) evalúa la eficacia con la que una economía utiliza su inventario de recursos. Este índice se basa en dos grupos de variables: operaciones y estrategias de las empresas y calidad del ambiente nacional de negocios.

El Índice de Competitividad para los Negocios, que es de nuestro interés particular por ser el que mide la competitividad empresarial, comprende dos grandes categorías, que se subdividen a su vez en numerosos indicadores: 
1. Operaciones y Estrategias de las Empresas, en esta categoría se incluyen: sofisticación del proceso de producción, naturaleza de la ventaja competitiva, alcance de la capacitación de los funcionarios, extensión del marketing, disposición a delegar autoridad, capacidad de innovación, gasto en investigación y desarrollo, presencia de cadena de valor, amplitud de los mercados internacionales, grado de orientación al consumidor, control de la distribución internacional, extensión de la política de marcas, confianza en la administración profesional, extensión de la compensación por incentivo, extensión de las ventas regionales, frecuencia del licenciamiento de tecnología extranjera.

2. Ambiente Nacional de Negocios, incluye cuatro indicadores fundamentales, que a su vez se dividen en otros.

a. Condiciones de Factores, contempla:

i. Infraestructura física: calidad de la infraestructura en general; calidad de la infraestructura férrea, portuaria, de transporte aéreo y de teléfono/fax; calidad del suministro eléctrico; teléfonos celulares por cada 100 habitantes; usuarios de Internet por cada 100 habitantes.

ii. Infraestructura Administrativa: protección de los negocios, independencia judicial, suficiencia del recurso legal en el sector público, carga administrativa para los nuevos emprendimientos, extensión de los trámites burocráticos.

iii. Recursos humanos: calidad de las escuelas de negocios, escuelas públicas y educación en matemática y ciencias. 
iv. Infraestructura tecnológica: patentes per cápita, disponibilidad de científicos e ingenieros, calidad de las instituciones de investigación científica, colaboración para la investigación entre la universidad y la industria, protección de la propiedad intelectual.

v. Mercados de capital: sofisticación del mercado financiero, disponibilidad de capital de riesgo, facilidad de acceso a préstamos, acceso al mercado local de valores.

b. Condiciones de la demanda: sofisticación de los compradores, adopción de productos nuevos por parte de los consumidores, adquisición gubernamental de productos de tecnología avanzada, presencia de estándares que regulan la demanda, leyes relacionadas con la tecnología de la información, rigor de las regulaciones ambientales.

c. Industrias Relacionadas y de Apoyo: calidad de los proveedores locales, estado de desarrollo de conglomerados (clusters) de empresas, disponibilidad local de maquinaria y de servicios especializados de investigación y capacitación, extensión de la colaboración en productos y procesos, cantidad de proveedores locales, disponibilidad local de componentes y partes.

d. Contexto para la Estrategia y Competencia de las Empresas, incluye:

i. Incentivos: extensión de subsidios gubernamentales que distorsionan, favoritismo en las decisiones de los funcionarios de gobierno, cooperación en las relaciones entre trabajadores y empleadores, eficacia de las autoridades corporativas. 
ii. Competencia: liberalización de barreras comerciales ocultas, intensidad de la competencia local, extensión de los competidores de base local, efectividad de la política anti monopólica, descentralización de la actividad corporativa, costos de actividades ilegales de otras empresas, liberalización arancelaria

Cuatro aspectos constituyen la crítica fundamental a la base teórica de Porter (1990), que sustenta este índice:

- El modelo presenta un defecto al no incorporar propiamente la actividad de las multinacionales, además de no reconocer la necesidad de un diamante doméstico para una nación y otro externo de sus relaciones internacionales como con los socios comerciales tal como se considera en algunos trabajos. (Rugman 1991, 1-64), (Dunning 1993; 135), (Moon, Rugman y Verbeke 1995, 114).

- Para otro grupo se considera que el modelo no funciona para las economías pequeñas 0 subdesarrolladas y es expuesto en trabajos realizados por otro autores (Bellak y Weiss 1993; 109-118), (Cartwirght 1993; p.68), ( Cho y Moon 2000; 106). De acuerdo con los autores para mitigar las limitaciones del modelo de las ventajas competitivas se deberían restaurar los factores determinantes de la competitividad de las naciones, de modo que se pueda resaltar la importancia de los factores humanos, que son los que conducen a la economía de los países a una mejor competitividad internacional.

- Existe un grupo de teóricos que han detectado una falta de precisión a la hora de definir conceptos, 
además de cierta subjetividad a la hora de definir los clúster existentes en sectores competitivos dentro del país como se ha afirmado algunos autores (Ryan 2004; 114), (Grant 1991; 535). Este último en su artículo publicado en "Estrategic Management Journal" evalúa el libro de Porter y afirma que existen otros factores además de la sofisticación tecnológica, las capacidades y las relaciones con clientes, para general ventajas competitivas. Este autor critica lo que para Porter puede suponer ventajas o desventajas de algunos de los factores son ventajas o viceversa en otros. Por último se señala que la relación causa y efecto entre los elementos que conforman el diamante tampoco está claro.

- Por último, Porter olvida el análisis la MIPYMES y su importancia en la economía global y doméstica. Las tendencias mundiales actuales en el campo empresarial reconocen en las MIPYMES una parte fundamental de la economía de cualquier nación. Los datos revelan que más del $90 \%$ del universo de empresas formales e informales en los distintos países se ubican en este grupo, participando también de forma muy elevada en las ventas totales, las exportaciones, el PIB y el empleo. Cualquier dificultad general en estas organizaciones menores repercute desfavorablemente en los indicadores macroeconómicos y sociales del país de que se trate

\section{Las MIPYMES en México}

Para México las MIPYMES, constituyen una parte determinante de la estructura económica del país, por su alto impacto en la generación de empleos y en la 
producción nacional. Los datos estadísticos del INEGI, señalan que existen aproximadamente 58millones $114 \mathrm{mil}$ 56 entidades empresariales, de las cuales $99.8 \%$ constituyen MIPYMES y ocupan $78.5 \%$ de todo el personal, pero sólo participan en la generación del $50 \%$ del PIB.

\section{Tabla No.1}

Las MIPyMEs en México (2009)

\begin{tabular}{|c|c|c|c|c|}
\hline Concept & Total & $\begin{array}{c}\text { Porcentaj } \\
\text { e }\end{array}$ & $\begin{array}{c}\text { Personal } \\
\text { Ocupad } \\
\text { o }\end{array}$ & $\begin{array}{l}\text { Porcentaj } \\
\text { e }\end{array}$ \\
\hline Empresas & $\begin{array}{c}514405 \\
6\end{array}$ & 100 & $\begin{array}{c}2772740 \\
6\end{array}$ & 100 \\
\hline $\begin{array}{c}\text { Microempres } \\
\text { as }\end{array}$ & $\begin{array}{c}489714 \\
1\end{array}$ & 92.5 & $\begin{array}{c}1264369 \\
7\end{array}$ & 45.6 \\
\hline $\begin{array}{l}\text { Pequeñas } \\
\text { Empresas }\end{array}$ & $\begin{array}{c}221194 . \\
4\end{array}$ & 4.3 & 6599123 & 23.8 \\
\hline $\begin{array}{l}\text { Medianas } \\
\text { Empresas }\end{array}$ & 15432.1 & 0.3 & 2523193 & 9.1 \\
\hline $\begin{array}{l}\text { Grandes } \\
\text { Empresas }\end{array}$ & 10288.1 & 0.2 & 5961393 & 21.5 \\
\hline Mujeres & & & $\begin{array}{c}1178414 \\
7\end{array}$ & 42.5 \\
\hline Hombres & & & $\begin{array}{c}1594325 \\
9 \\
\end{array}$ & 57.5 \\
\hline
\end{tabular}

Fuente: INEGI. Censo Empresarial 2009

Las estadísticas del INEGI, muestran la existencia de un proceso piramidal de la estructura empresarial en México. En nuestro país el $95.2 \%$ son microempresas, que ocupan al $45.6 \%$ de todo el personal, en tanto que las pequeñas empresas son $4.3 \%$ y emplean al $23.8 \%$; por último las 
medianas empresas son el $0.3 \%$ y ocupan al $9.1 \%$ del personal disponible, y el restante $0.2 \%$ son empresas de gran tamaño que emplean al $21.5 \%$ de las personas reportadas.

Entre los aspectos significativos mostrados por el Censo, se constata que en genero las mujeres representan el $42.5 \%$ del personal ocupado, mientras los hombres son el $57.5 \%$. Los estados que emplean mayor trabajo femenino son Tlaxcala con el 47\%; Oaxaca con 46.9\%; Morelos con $46.7 \%$, y Michoacán con $46 \%$.

Las actividades económicas, en nuestro país, se agrupan de la forma siguiente: $47.1 \%$ empresas de servicios, $26 \%$ empresas de comercio, $18 \%$ empresas de la industria manufacturera, el resto de las actividades representan el $8.9 \%$. Lo que muestra una mayoría de empresas dentro del sector terciario; mientras que la actividad manufacturera tiene una participación de solo el $18 \%$ que se agrupan fundamentalmente en los estados de Chihuahua con $40.6 \%$, Baja California con 37.6\%, Coahuila con $34 \%$, Tamaulipas con $33.5 \%$ y Querétaro con $30.4 \%$.

\section{Problemas que enfrentan las MIPYMES en México}

Una de las características fundamentales de la economía mexicana es su orientación a la exportación por la estrechez de su mercado doméstico; sin embargo los datos muestran que no existe una correspondencia entre 
el modelo exportador y la participación de las MIPYMES en la actividad exportadora del país.

De acuerdo a las estadísticas planteadas en México existen 399 empresas grandes que concentran el $70.8 \%$ de los ingresos por concepto de exportación, mientras que las MIPYMES solo representan el $28.8 \%$ donde de este porcentaje, la mayor representatividad la tienen las medianas empresas. Por tanto si consideramos al crecimiento de México como una variable dependiente del funcionamiento del mercado externo y en especial de mercado de los Estados Unidos, los programas de la Secretaría de Economía para el fomento de la actividad de las MIPYMES, debe ser revisado y concretar planteamientos más específico que puedan ayudar de forma más eficiente al emprendedor mexicano.

Los principales problemas que afectan a la MIPYMES en México, están determinados por variables exógenas y endógenas al sistema económico, que pueden resumirse en: marco jurídico institucional; acceso y funcionamiento del mercado de factores productivos; acceso $y$ funcionamiento al mercado de bienes y servicios; gestión y cooperación empresarial y otros de carácter particular que están determinados por la región, la integración de familia, etc.

Un análisis del marco jurídico institucional de los negocios en México, revela que si bien ha quedado superado, en teoría, aspecto de importancia como lo constituye el marco regulatorios competitivo, como resultado de la nueva "Ley de Competencia"; la falta de reformas estructurales que deben llevar a una modificación puntual del marco jurídico institucional siguen siendo una 
afectación directa en la búsqueda del incremento de su competitividad.

Como consecuencia de lo anterior el acceso al mercado de factores productivos muestras limitaciones, que se acentúa por el pobre nivel para lograr créditos bancarios, las estadísticas indican que la banca privada, para realizar prestamos debe contar con una gran cantidad de garantías, lo que resulta una situación difícil cuando las MIPYMES en México, que no cuentan, en la generalidad, con locales propios; además de los problemas de contabilidad que poseen estos tipos de empresas que las sitúan ante la banca como instituciones no sujetas a crédito. Por lo tanto las MIPYMES tienen poca posibilidad de acceso a un mercado de trabajo donde exista la fuerza de trabajo calificada, que permita lograr los niveles de competitividad exigidos a nivel internacional y con la calidad requerida.

Los aspectos mencionados le condicionan a la MIPYMES en México, en muchos casos, una dificultad en el acceso al mercado de bienes al no contar con el aparato logístico, aunque cabe señalar que se han creado herramientas para que este tipo de empresa tenga acceso a ser proveedoras de las empresas paraestatales y organismos de gobierno.

Por último en las MIPYMES en México, se presenta una falta de cooperación y un predominio del individualismo, este proceso crea aislamiento y dificultad la búsqueda de economías de escalas. 
Estos problemas hacen que se tenga que trabajar en concreto en direcciones que permitan una mayor participación de la MIPYMES, entre ellas pueden citarse:

- Análisis más detallados del comportamiento de los mercados globales y nacionales no solamente desde la óptica de la macroeconomía en general, sino también mediante el estudio de aspectos macroeconómicos del comportamiento de los sectores

- Mejor análisis de la fuentes de financiamiento de las MIPYMES y los procesos de análisis de cobertura de riesgo, siguiendo los lineamientos establecidos por los Acuerdo de Basilea de tal forma que existan criterios adecuados para que las empresas cuentes con los recursos financieros que le den acceso al mercado de factores.

- Desarrollar programas de capacitación y entrenamiento de los trabajadores empelados en las MIPYMES de tal forma que estas instituciones tengan un personal ocupado con mayor flexibilidad y eficiencia para el desarrollo de las actividades.

- Los dos aspectos anteriormente expuestos permitirán que las MIPYMES, se encuentren en condiciones de tener un mejor acceso a la tecnología y mejorar su logística en acceso al mercado de bienes y servicios.

- Además es necesario fomentar el mejoramiento de las formas de gestión y colaboración para que este tipo de empresa pueda lograr encomias de escalas en un mundo altamente competitivo.

Estos aspectos crean la necesidad de desarrollar una forma de medición de competitividad para este tipo de empresa que le permita accionar de manera adecuada y 
rápida sobre los factores que deben permitirle aumentar su eficiencia.

\section{Metodología para Índice Ibero de Competitividad Empresarial (IICE)}

La competitividad empresarial es definida como la capacidad que tiene una organización de mantener sistemáticamente ventajas competitivas que le permitan alcanzar, sostener y mejorar una determinada posición en el mercado. Más aún, se puede decir que es hacer las cosas mejor que los demás.

De esta manera, la competitividad está directamente relacionada con la forma de planear y desarrollar cualquier iniciativa de negocios. Cuando el empresario se toma el tiempo necesario para planear, esto es, establecer metas y objetivos, diseñar programas para alcanzarlos y realizar la evaluación para mantener al día los planes, en fin, para tener el control, está identificando las oportunidades que le permiten ser proactivo y ser más competitivo.

La ventaja competitiva de una empresa, es decir, los conocimientos económicos, financieros, tecnológicos y humanos de los que dispone, los mismos de los que carecen sus competidores o que estos tienen en menor medida, hace posible la obtención de unos rendimientos superiores a la competencia. Esta habilidad se origina en esa capacidad de planear y organizarse.

La competitividad independiente de sus mediciones macros, se genera en la empresa como unidad 
determinante en el empleo de los recursos productivos. Partiendo del criterio de competitividad adoptado, su medición es un complejo proceso en el cual hay que tener la mayor amplitud posible. El modelo, que presentamos es un modelo de corto plazo, donde el índice IBERO de competitividad de las MIPYMES, es una variable dependiente de:

$I C E=f(G ; G e ; G f)+(Q+T)+M e+A e$

En donde:

G; Gestión Empresarial

Ge; Gestión de la Comercialización

Gf; Gestión Financiera

Q; Producción

T; Tecnología

Me; Entorno o factores exógenos relacionados con la meta economía

Ae; Entorno o factores exógenos relacionados con el ambiente ecológico

Para la conformación del modelo se adoptan las siguientes definiciones:

- Gestión empresarial como la actividad empresarial que a través de diferentes individuos especializados, como ser: directores institucionales, consultores, productores, gerentes, entre otros, y de acciones, buscará mejorar la productividad y la competitividad de una empresa o de un negocio.

- Gestión de comercialización es el conjunto de actividades desarrolladas con el fin de facilitar la venta de una mercancía o un producto.

- Gestión financiera se consideran como todos los procesos que consisten en conseguir, mantener y utilizar dinero, sea físico (billetes y monedas) o a través de otros instrumentos, como cheques y tarjetas 
de crédito. La gestión financiera es la que convierte a la visión y misión en operaciones monetarias.

\section{Esquema gráfico de construcción del IICE}




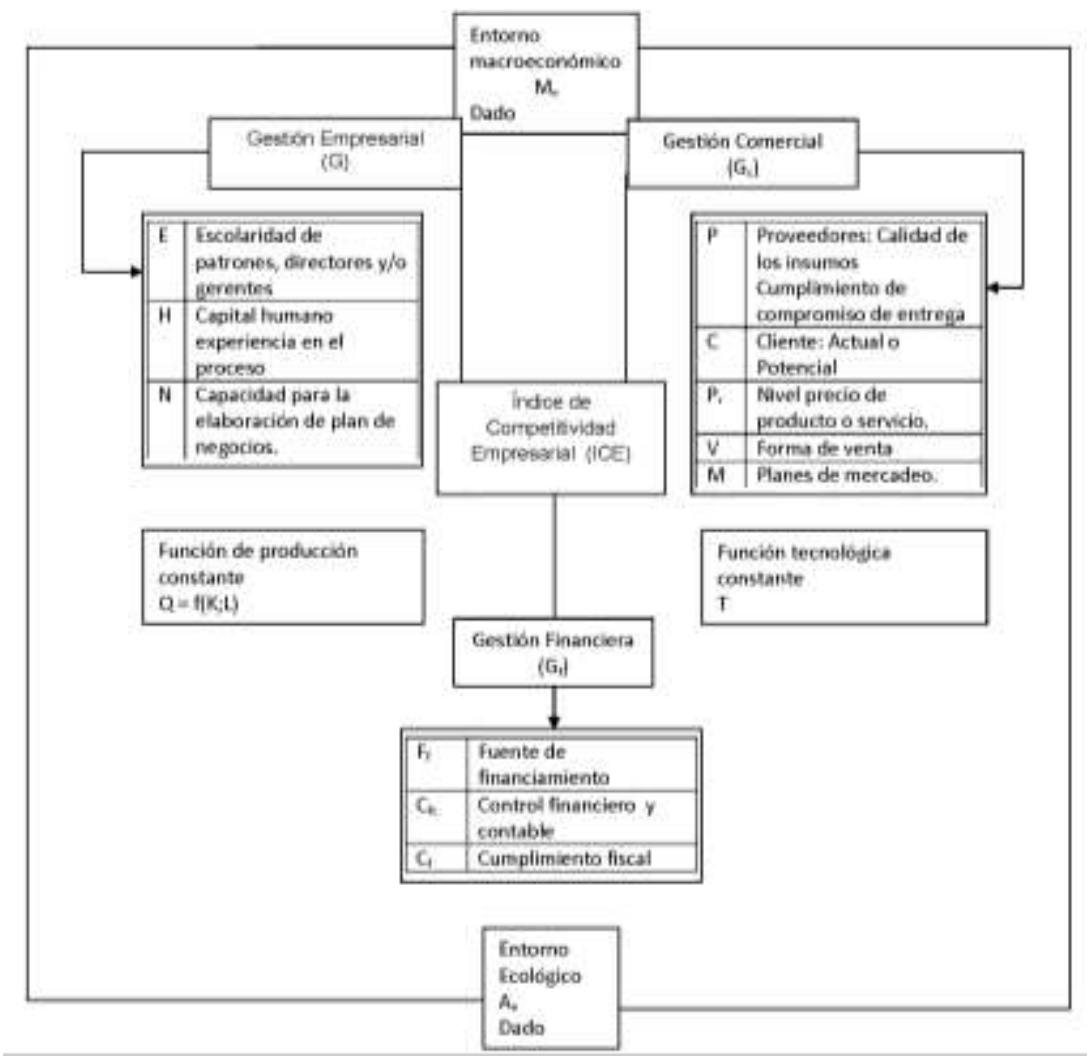

Para el modelo de corto plazo propuesto, la función de la producción y la tecnología se considera constante, justificado en que las modificaciones en estas variables no son de forma inmediata ya que el empresario generalmente responde a cambios en la demanda de mercado, ante la ausencia de una cultura de innovación constante. Razón por cual solamente se enuncian. Q; Ceteris Paribus

T; Ceteris Paribus. 
La competitividad se da en el marco de un entorno macroeconómico y ambiental que se considera inalterable en el corto plazo, razón por la cual las consideramos como variables exógenas dadas. Estas variables hacen referencias a los grandes objetivos económicos del país en materia fiscal y monetaria, materializados en las políticas gubernamentales y que inciden en la competitividad empresarial, al igual el medio ambiente, que es de una gran incidencia en la competitividad empresarial y en la formulación de sus estrategias de sustentabilidad.

Este índice debe cubrir dos aspectos fundamentales:

- Debe ser representativo del tamaño y capacidad de la empresa en su totalidad

- Debe ser comparable respecto al tiempo y al espacio

El índice se propone generarse a partir del uso del rango percentil ${ }^{1}$, dado que tiene como ventaja en la interpretación de los resultados: el valor del rango percentil para una empresa, nos indica el porcentaje de empresa respecto del cual es más competitiva, además de ser muy sencillo ante la no complejidad de variables Una vez que todos los datos se uniforman, es necesario integrarlos o sintetizarlos en un solo índice. Para la

\footnotetext{
${ }^{1}$ El percentil es una medida de posición no central que nos dice cómo está posicionado un valor respecto al total de una muestra. También se usan los cuartiles, deciles o quintiles pero no son más que casos particulares.

El concepto es sencillo. Si tenemos una muestra con muchos valores y la dividimos en 100 partes, cada una de ellas es un percentil. El percentil está referenciado de 0 a 100 . El Percentil 0 es el menor valor de la muestra y el Percentil 100 el mayor valor, técnicamente es el percentil iésimo, donde la i toma valores del 1 al 100. El i \% de la muestra son valores menores que él y el 100-i \% restante son mayores. Es decir, supongamos que tenemos una muestra con 1000 datos de personas y salarios. El P-75 sería el valor de salario que ganan el 75\% de las personas, o el P-20 el que ganan el $20 \%$
} 
realización de lo anterior se pueden emplear tres métodos:

- promedio simple,

- promedio ponderado,

- análisis de componentes principales.

El análisis de componentes principales es un método estadístico que asigna las ponderaciones a partir de la información contenida en los datos. Surgen sin embargo dos dificultades:

- La primera es cómo validar e interpretar tales ponderaciones. Siendo ésta una de las primeras versiones del Índice no hay información disponible para validar tales ponderaciones. Una opción sería presentar las ponderaciones a un "panel de expertos" pero ello generaría nuevas complicaciones metodológicas.

- La segunda es cómo interpretar esas ponderaciones. Con frecuencia, alguna de las ponderaciones tendrá valor negativo, surgiendo la cuestión de cómo un factor de competitividad puede restar a la misma en lugar de sumar.

El promedio ponderado requiere establecer los valores con los cuales ponderar los subíndices y los factores. Esto plantea la cuestión de cómo eliminar la subjetividad en la determinación de los pesos de ponderación.

A fin de asegurar la simplicidad de cálculo y de garantizar la objetividad del procedimiento se utilizará el método del promedio simple. Donde se establece que durante los próximos tres años deberán hacerse estudios estadísticos para determinar si es conveniente utilizar alguno de los otros dos métodos de agregación. 
La forma de cálculo la expresamos de la siguiente forma:

Paso I. Para cada empresa, se calcularan los valores de los subíndices, aplicando rangos percentiles a los valores de la variable correspondiente.

El rango percentil de la variable $k$ para la empresa $i$ se calculará:

Rango percentil $k, i=$ (Número de variables menores a la variable k,i / Número de empresas -1)

Si la variable $k, i$ es la mayor de todas las variables $k$, entonces el rango percentil es igual a 1 . Si la variable $k, i$ es la menor de todas las variables $k$, el rango percentil es igual a 0 . En consecuencia el valor de los subíndices fluctúa entre 0 y 1.

A fin de asociar el mayor valor de la variable con un mejor resultado en el índice, se asociará un valor "1" al mejor resultado de cada subíndice.

Paso II. Para cada empresa, cada uno de los factores es calculado como el promedio simple de los subíndices que componen el factor.

Paso III. Para cada empresa, el Índice de Competitividad Empresarial se obtendrán como el promedio simple de los factores obtenidos en el paso anterior

Paso IV. Se ordenan los valores del Índice de Competitividad Empresarial para obtener el ranking de las empresas. 
En los casos en que varias empresas comparten un mismo valor, se considera un empate y se asigna a todas esas regiones la misma posición.

\section{Conclusiones}

La operación de las micro y pequeñas empresas constituye un fuerte impulso a la creación de empleo y de aumento de ingresos de amplios segmentos de la población, constituyendo un gran soporte en el desarrollo económico y social, con un gran impacto en todo el territorio nacional. Estas empresas son importantes porque apuntalan el crecimiento de los mercados. Por su gran impacto en el desempeño económico y social es necesario profundizar en el conocimiento de las micro y pequeñas empresas pues su éxito es parte del éxito del país.

El papel que las PYMES juegan en la economía es preponderante y de gran trascendencia, dado que significan una respuesta al desempleo, propician una mejoría en los ingresos y por ende tienden a elevan la calidad de vida de las familias. En la actualidad, las micro y pequeña empresas son las de mayor importancia en nuestra economía, Esta realidad implica un reto mayor que debe ser enfrentado mediante su incorporación a los beneficios de la apertura comercial, particularmente a través de un incremento en sus índices de competitividad. Sin embargo entre los aspectos que han limitado la elevación de la productividad y eficiencia de las empresas de menor tamaño, destacan las siguientes:

- Limitaciones de capital y de acceso a créditos favorables, dada la debilidad del sistema financiero y 
en especial de la banca de desarrollo, para apoyar eficientemente a la planta productiva;

- dificultades para incorporar tecnología avanzada, acentuada por la desvinculación con las universidades y centros de investigación que pudieran coadyuvar a ello;

- desvinculación y aislamiento con respecto a otras unidades productivas, cancelando la posibilidad de obtener mejores costos de producción; y,

- restricciones para acceder a servicios especializados de asesoría y consultoría para superar problemas de diversa índole: técnicos, jurídicos, administrativos, financieros o comerciales, que desvían gran parte del tiempo del empresario; así como dificultades para acceder a la información confiable y oportuna sobre las posibilidades que ofrecen otros mercados, e inhiben el conocimiento de programas institucionales de apoyo a la competitividad.

La problemática descrita busca responderse con la instrumentación de parámetros y medidas económicas y financieras sencillas, que permitan generar la información necesaria para la toma de decisiones, incorporando además prácticas promotoras de comunicación. Esto responde a los vicios observados en la gestión de la microempresa, donde los dueños no separan los recursos financieros de la empresa de los propios, no llevan registros simples de contabilidad, carecen de estructuras organizativas, no son sujetos de crédito de la banca comercial, y no promueven ni comercializan adecuadamente sus productos. Índice será un instrumento que ayude a superar estas situaciones

\section{Fuentes de Consulta}


- BELLAK, C. J. \& Weiss, A., (1993), A note on the Austrian "Diamond, Management International Review", Vol.33, p.109-118.

- CARTWRIGHT, W. R., 1993, Multiple Linked "Diamonds" and the International Competitiveness of Export-Dependent Industries: The New Zealand Experience, Management International Review, Vol.33, p.55-70.

- CHO, D. S., \& Moon, H. C., 2000, From Adam Smith to Michael Porter. Singapore: World Scientific.

- DUNNING, J.H. (1992) The competitive advantage of countries and the activities of transnational corporations. Transnational Corporations, 1 February, No. 1, 135- 68

- http://www.observatoriopyme.org/encuestas-yestudios/cifras-de-pymes/

- GRANT, Robert M. (1991) "Porter's Competitive Advantage of Nations': An assessment". Estrategic Management Journal Oct 1991. 12,7, ABI/INFORM Global, p.535.

- MOON, H.C., Rugman, A.M., \& Verbeke, A. (1995). The generalized double iamond approach to international competitiveness. In A. Rugman, J. Van Den

- BROECK \& A. Verbeke (Eds.), Research in global strategic management: Volume 5: Beyond the diamond (pp. 97-114).

- PORTER. M. E (1990) "The Competitive Advantage of Nations. Free Press, New York, p 19 
- RUGMAN, A.M. (1991), Diamond in the rough. Business Quarterly. 55(3), 61-64.

- RYAN C y Phillips P. (2004) Knowledge management in advanced technology industries: An examination of international Agricultural Biotechnology clusters. Enveronment and planning C, Government an Policy. Londres UK.

- OIT Oficina Regional para América latina y el Caribe. Víctor Tokman Director dela Oficina Regional de la OIT para América Latina y el Caribe. (2002) "De la informalidad a la modernidad"

- Organización para la Cooperación y el Desarrollo Económico (OCDE), (2004). "Promoting entrepreneurship and innovative SMEs in a global economy. Towards a more responsible and inclusive globalization". Second OCDE Ministerial Conference on SMEs, 2-5 junio, Estambul. OCDE (2002) Small and Medium Entreprise Outlook.2002, The Bologna Charter on SME Policies, París, OCDE, 2000 